\title{
What's the point? Infants' and adults' perception of different pointing gestures
}

\author{
Ebru Ger ${ }^{1}$, Stephanie Wermelinger ${ }^{1}$, Maxine de Ven ${ }^{1}$, and Moritz M. Daum ${ }^{1,2,3}$ \\ ${ }^{1}$ Department of Psychology, University of Zurich, Switzerland \\ ${ }^{2}$ Center for the Interdisciplinary Study of Language Evolution (ISLE) Zurich, Switzerland \\ ${ }^{3}$ Jacobs Center for Productive Youth Development, University of Zurich, Switzerland
}

\begin{abstract}
Author Note
Working paper. Please do not quote or cite without the authors' permission.

Ebru Ger (iD https://orcid.org/0000-0002-6140-1807

Stephanie Wermelinger (D) https://orcid.org/0000-0002-6500-9108

Moritz M. Daum (iD https://orcid.org/0000-0002-4032-4574

The authors have no conflict of interest to disclose. This study was approved by the local ethics committee. We used materials from Bertenthal et al. (2014) with their permission. This study was preregistered (see link:

https://doi.org/10.17605/OSF.IO/HB6ZP). All data, analysis code, and research materials have been made available in OSF at http://doi.org/10.17605/OSF.IO/F3EK5.

Correspondence concerning this article should be addressed to Ebru Ger, University of Bern, Institute of Psychology, Fabrikstrasse 8, 3012 Bern, Switzerland. E-mail:
\end{abstract} ebruger@gmail.com 







\section{What's the point? Infants' and adults' perception of different pointing gestures}

Following the pointing gestures of others is an essential ability infants need to develop for successful communication and social interaction. This ability has long been suggested to emerge around infants' first birthday (Carpenter et al., 1998; Daum et al., 2013; Deák et al., 2000; Leung \& Rheingold, 1981; Morissette et al., 1995). However, recent studies using different measures documented orienting the attention in the direction of a pointing hand in infants younger than 12 months, and as early as 4 months of age (Amano et al., 2004; Bertenthal et al., 2014; Gredebäck et al., 2010; Melinder et al., 2015; Rohlfing et al., 2012). Several key features seem to determine whether infants successfully follow pointing: accompanying communicative speech (Daum et al., 2013), accompanying gaze (Amano et al., 2004; but see von Hofsten et al., 2005), familiarity of the pointer (Amano et al., 2004), humanness of the pointing gesture (Bertenthal et al., 2014; Schmitow et al., 2016), clear unidirectionality of the pointing gesture (Lakatos et al., 2009; Schmitow et al., 2016), and the movement of the pointing hand (Rohlfing et al., 2012). However, one important potential feature, the shape of the pointing hand, has not been investigated concerning point following in infants.

A few studies with adult participants showed that they orient faster to pointing with the canonical index-finger shape than other shapes such as the fist or the pinky finger (Ariga \& Watanabe, 2009; Daum \& Gredebäck, 2011b; Nishimura \& Michimata, 2013). These studies employed a spatial cueing paradigm, where participants' orientation was determined by their manual reaction times gauged by key presses. A cueing effect - faster reactions to targets that appear in the direction cued by the pointing than those that appear in the uncued direction - emerged for all hand shapes but a particularly larger cueing effect was shown for the index-finger shape. This suggests that adults' orientation to pointing gestures (henceforth referred to as pointing perception) is not only based on the directionality of a pointing hand but also its form. Adults' pointing perception may be tuned in to the social relevance of the canonical index-finger pointing shape. 
The following two aims guided the present study. First, we aimed to replicate previous cueing effects with adults for the index and pinky finger and extend it to the whole hand. Whole-hand pointing is used by adults (Cochet \& Vauclair, 2014; Flack et al., 2018), however, to our knowledge, its perception has not been investigated before. For this, we used a spatial cueing paradigm on an eye tracker to capture participants' orientation of attention by their gaze shifts. We expected adults to similarly show a cueing effect for all hand shapes. We furthermore expected the cueing effect to be larger for index-finger pointing reflecting particular sensitivity to the index finger compared to other forms of pointing.

The second aim was to investigate, using the same spatial cueing paradigm, whether such increased sensitivity to the canonical index-finger pointing already exists in infancy. The spatial cueing paradigm has been successfully applied in revealing infants' earliest abilities to orient their attention toward the direction of a cue (Bertenthal et al., 2014; Rohlfing et al., 2012). For exploratory reasons and because we tested the infants' parents as our adult sample, we additionally examined whether and to which extent parents' and their infants' general processing speed and cueing by pointing gestures were related.

In infancy, the shape of a pointing hand has been examined in relation to the production of pointing. There is evidence that the index-finger shape is a critical feature in the production of pointing in infancy. Infants start pointing between 7 to 15 months of age (Camaioni et al., 2004; Carpenter et al., 1998; Leung \& Rheingold, 1981; Lock et al., 1990). They mostly start out by pointing with their whole hands and only later develop index-finger points (Ger et al., 2018; Kita, 2003; Liszkowski \& Tomasello, 2011; Lock et al., 1990; Lüke, Ritterfeld, et al., 2017). On average, index-finger pointing emerges at 11-12 months (Cameron-Faulkner et al., 2015; Lüke et al., 2019; Rüther, 2019). Infants' index-finger pointing is related to important aspects of their communicative and social-cognitive development. For example, at 12 months, infants who pointed with the index finger were better at understanding others' communicative intentions, pointed more 
frequently, and vocalized along with their points more often compared to their counterparts who pointed only with the whole hand (Liszkowski \& Tomasello, 2011). This distinction has also been indicative of later language delays. Twelve-month-olds who only pointed with their whole hands but never with the index finger were more likely to develop a primary language delay one year later (Lüke, Grimminger, et al., 2017). In sum, hand shape plays a role in the production of pointing, especially at 12 months. However, we do not know whether it also plays a role in the perception of pointing. Studies examining pointing perception with infants so far always focused on index-finger pointing. There is a great deal of evidence that infants perceive index-finger pointing as directed towards a location in space by 12 months (Carpenter et al., 1998; Daum et al., 2013; Deák et al., 2000), if not earlier (Bertenthal et al., 2014; Gredebäck et al., 2010; Melinder et al., 2015; Rohlfing et al., 2012). Hence, taken together with the literature on pointing production, 12 months seems to be the crucial age to study pointing perception and the potential role of hand shapes. Therefore, we tested infants aged 12 months and examined (1) whether they orient to pointing with different hand shapes, and (2) whether they are more sensitive to orienting to pointing with the index finger compared to pointing with the whole hand and the pinky finger. The results of the current study are expected to shed more light onto the origins of pointing perception.

In our view, infants' pointing perception may be influenced by different aspects such as their social and motor development - what they have learned from adults and how they point themselves - as well as the affordance of the form of pointing - the directionality or distinctness of the pointing.

In the social domain, we may consider two mechanisms. First, infants might be learning to follow pointing by observing adults' pointing. Thereby, they understand the communicative motivation behind adults' pointing and imitate its functional use (Cochet \& Vauclair, 2010). A number of studies document associations between caregiver and infant pointing (Liszkowski \& Tomasello, 2011; Rowe \& Goldin-Meadow, 2009), some of which 
119

suggest a causal (or at least directional) effect of caregiver pointing on infant pointing (Rowe and Leech, 2019; Salomo and Liszkowski, 2013; but see Matthews et al., 2012 for a lack of causal relation). There is also evidence suggesting that infants are socialized into the canonical index-finger pointing shape through their social context. For example, at 14 through 28 months, sighted children are documented to point more often with their index finger than the whole hand whereas congenitally blind children pointed predominantly with their whole hand and almost never with the index finger (Iverson et al., 2000). Under this view, we would expect infants to only orient or orient faster to the canonical index-finger pointing of adults than the much less frequently used whole hand and pinky finger shapes. Note that the canonical hand shape of pointing is with the index finger, at least in the European country where our data comes from.

Second, infants might be learning to follow pointing mainly by observing adults' reactions to their own pointing in social interactions (Leung \& Rheingold, 1981; Moore \& Corkum, 1994). In line with this, infants' pointing frequency at 10 months was found to be associated with their point-following abilities at 12 months (Ger et al., 2018). Yet, even at 12 months, there is considerable variation in the hand shape with which infants point. Infants point about half the time with the index finger and half the time with the whole hand on average (Ger et al., 2018; Liszkowski \& Tomasello, 2011; Lüke, Ritterfeld, et al., 2017), and at least half of the infants point with both the index finger and the whole hand (Liszkowski \& Tomasello, 2011; Lüke, Ritterfeld, et al., 2017). At the same age, parents' responsiveness to their infants' points does not differ between index-finger and whole-hand points (Ger et al., 2018; Lüke, Ritterfeld, et al., 2017). Therefore, at this age, infants may still orient equally fast to whole-hand and index-finger pointing. They may not orient at all or orient slower to the pinky-finger pointing because it is not in their repertoire.

In the motor domain, infants' ability to produce a certain hand shape for pointing may be directly linked to the way they perceive others' pointing. Research on the processing of actions suggests that the ability to perceive actions as goal-directed is 
influenced by the observers' ability to perform these actions (infants: Ambrosini et al., 2013; Melzer et al., 2012; Sommerville et al., 2005, adults: Calvo-Merino et al., 2006;

Diersch et al., 2012). Furthermore, action perception and production develop hand in hand (Daum et al., 2011; Daum et al., 2013; Gampe et al., 2015; Wermelinger et al., 2018).

Transferring these findings to the development of pointing gestures suggests that infants at the age of 12 months might orient equally fast to both the index finger and the whole-hand shape, and not orient or orient slower to the pinky-finger shape. Because, at 12 months, infants still point both with the index finger and the whole hand, while pointing with the pinky finger is not in their repertoire.

Furthermore, the affordance of the pointing shape may influence how fast infants orient to the different pointing shapes. If infants are cued by the directionality of the gestures (Lakatos et al., 2009; Schmitow et al., 2016), then they would be equally fast to orient to all three hand shapes, because they are all directional. If infants are influenced by the distinctness of the pointing form, we would expect them to orient equally fast to the index-finger and the pinky-finger point, and faster than the whole-hand point. Because, both the index and the pinky finger shapes provide a more distinct line of regard for the recipient to follow compared to the whole hand.

In summary, in light of these possible mechanisms, we formulate the following hybrid hypotheses. If infants' own repertoire of pointing use is the main driving force for their pointing perception (either through motor coupling of production and perception or through the adult responses they receive for their own pointing, or both), then they should be equally good in following index-finger and whole-hand pointing. If observational learning is the main driving force, then infants should be better in following index-finger pointing. If the attention-directing affordance of the pointing shape is the main driving force, then infants should either follow all shapes equally well (directionality) or be better in following index-finger and pinky-finger pointing (distinctness). Because index-finger pointing cuts across all three possible mechanisms - as it is in the repertoire of infants, the 
conventional form of adult pointing, and a distinct indicator of directionality - we believe that infants may still show a greater sensitivity to following index-finger pointing.

\section{Method}

\section{Transparency and Openness}

We report how we determined our sample size, all data exclusions, all manipulations, and all measures in the study. This study was pre-registered on osf (see link: https://doi.org/10.17605/OSF.IO/HB6ZP). All data, analysis code, and research materials have been made available in OSF at http://doi.org/10.17605/OSF.IO/F3EK5. Data were analyzed using R[version 4.0.1](R Core Team, 2021).

\section{Participants}

We based our sample size planning on Bertenthal et al. (2014). They tested 16 4-month-olds and 16 6-month-olds and achieved a power of .68 and .55 respectively to find a congruency effect with a 100ms-SOA. Considering a diminishing effect size with increasing age, and potential attrition, we planned a sample size of 40 infants. However, we later increased our sample size and used mixed effects models to be able to additionally take the trial-wise variation into account, which we did not foresee earlier. The results of the pre-registered ANOVA analysis on 55 infants can be found in the Appendix. Please note that the observed power for the congruency effect in this ANOVA analysis is $96 \%$.

We analyzed the data of 12-month-old infants $(n=55,28$ girls, 27 boys; $M=367$ days, $S D=9.28$ days) and their parents ( $n=63,60$ mothers, 3 fathers; $M=35.5$ years, $S D=3.67$ years). Additional 11 infants participated but were excluded from the analysis due to several reasons. One infant (girl) was younger than our target age, three infants (1 girl, 2 boys) fussed and therefore the eye-tracking task could not be performed, one infant's (boy) eye-tracking data could not be obtained due to technical problems, and six infants (4 girls, 2 boys) did not complete the minimum number of necessary trials. To be included in 
the analyses, participants (parents and infants) had to complete a minimum of 6 trials per stimulus cue (i.e., hand shape: index-finger, whole-hand, pinky-finger) by congruency condition (i.e., congruent, incongruent), totaling a minimum of 36 trials (based on Bertenthal et al., 2014). Two additional parents (mothers) participated but were excluded. One had to attend to her child and therefore could not perform the eye-tracking task, and the other did not complete the minimum number of necessary trials.

Contact information for the parents and their infants was obtained from public birth records. Parents were contacted by phone and e-mail. All infants were Swiss-German monolinguals. All infants had a normal birth weight $(>2,500 \mathrm{~g})$ and were born full-term (37-42 weeks of gestation), except for one infant who was born in the 35th week. This infant was nevertheless included because it was at the higher end of the age range (379 days old) at the measurement and did not show any signs of developmental delays. Furthermore, running the analyses without this child did not lead to a different result pattern. All parents gave informed consent before the study and all procedures were approved by the local ethics committee and performed in accordance with the ethical standards of the 1964 Helsinki declaration and its later amendments. Children received a small toy and a certificate after their participation.

\section{Test Environment, Stimuli, and Apparatus}

The laboratory was unfurnished except for the test equipment. The stimuli were presented, and gaze was measured from one eye using an 17-inch (800px, 600px) Eye Link 1000 eye tracker (Eyelink 1000Plus, SR Research, sample rate: $500 \mathrm{~Hz}$ ). The infants were seated in a car seat which was placed $50-55 \mathrm{~cm}$ in front of the eye tracker. During the eye-tracking assessment of the parents, they were seated in a chair in front of the eye tracker at the same distance.

At the beginning of the experiment, a video with drawings of animals was presented on the screen, while the experimenter adjusted the eye-tracking settings. Then, a 


\section{Figure 1}

Pointing gestures that were used in the spatial cueing paradigm as stimulus cues. From left to right: index finger pointing, pinky finger pointing and whole hand pointing. See the online article for the color version of this figure.
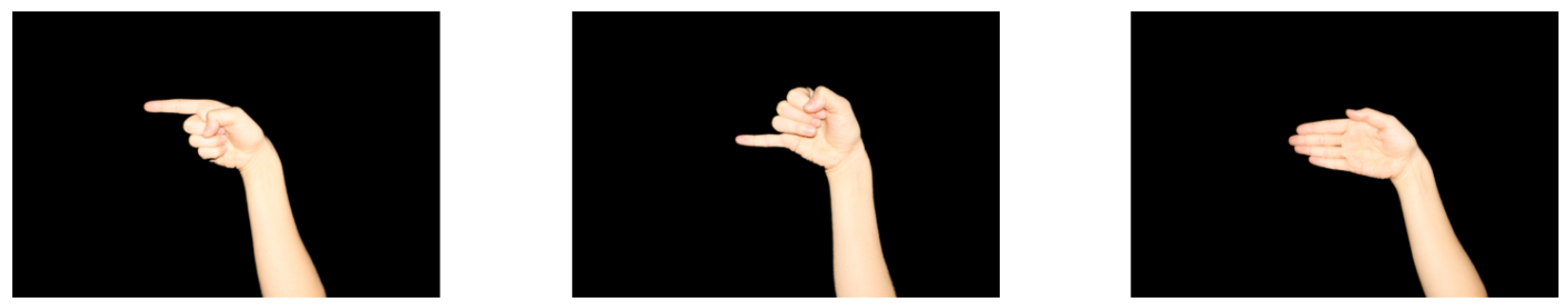

calibration was performed where a black and white disc expanded and contracted with an accompanying sound sequentially at the center and four corners of the screen, on a black background. After every sixth trial during the experiment, a drift check was performed to check if the calibration was still valid or a re-calibration was needed. A re-calibration was manually activated if necessary (drift check deviation $>1^{\circ}$ visual angle).

At the beginning of each trial, a static and gaze-contingent attention-getter, a colored pinwheel or a beach ball, appeared on the center of the screen subtending at $5.9^{\circ} \mathrm{x}$ $5.9^{\circ}$ visual angle without an accompanying sound. The stimulus cue also appeared in the center of the screen and was a pointing hand in one of three different hand shapes (see Figure 1). It vertically started at the bottom of the screen and was horizontally centered on the screen. Note that the center of the palm of the pointing hand (at 400px, 350px) was where the attention-getter was also previously centered at. The pointing gestures all included the hand in a horizontal position, and the forearm in a vertical position $\left(12.9^{\circ}\right.$, $13.5^{\circ}$, see Figure 2). All the pointing gestures oscillated back and forth $0.5^{\circ}$ at a rate of 3 Hz, following Bertenthal et al. (2014).

The target was one of 12 different videos containing a colorful toy animal picture that was moving around in a white rectangular region with an accompanying melodic sound $\left(4.6^{\circ}, 6.0^{\circ}\right.$, see Figure 2). The 12 target videos (from Bertenthal et al. (2014)) were 


\section{Figure 2}

Image of the pointing index finger and the target with visual angle dimensions. See the online article for the color version of this figure.



randomly selected for every trial within a block without replacement. The target was located on either side of the stimulus cue. The target's center was located $15.2^{\circ}$ to the left or right of the screen center. The target's midpoint was vertically aligned with the midpoint of the palm of the pointing hand.

The areas of interest (AOIs) for the target were defined around each target $5.5^{\circ}$ wide and $6.8^{\circ}$ high. The stimulus cue AOI was defined around the stimulus cue $14.5^{\circ}$ wide and $13.7^{\circ}$ high. The AOIs for the stimulus cue and target are indicated by the blue arrows around each of them in Figure 2. The AOIs were used to assess whether an infant or adult was looking at the defined area or not. 


\section{Design}

The spatial cueing paradigm consisted of 8 blocks, each containing 12 trials, leading to a total of 96 trials. The 12 trials within each block consisted of each combination of 2 directions (left and right), 2 congruencies (congruent and incongruent), and 3 hand shapes (index finger, whole hand, or pinky finger). The pointing cue was not informative for the target as it appeared on the congruent and the incongruent side an equal number of times. There were 4 different pseudo-randomized trial orders such that each variable (direction, congruency, hand shape) was not repeated more than two consecutive trials. The infants were assigned randomly to one of the 4 trial orders. The parents were assessed with the same trial order as their infant. Both infants and parents were tested with the same design.

\section{Procedure}

All infants were tested individually with at least one parent present. Each infant and their parent were escorted to a reception room. For approximately 10 minutes, the experimenter described the test procedure to the parent and handed them a consent form to sign while the infant was encouraged to play with the experimenter. In the first part of the study, the spatial cueing paradigm was presented to the infant. The parent sat behind the infant but faced away from the screen, and on a tablet filled out the Infant Behavior Questionnaire-Revised Very Short Form (IBQ-R VSF, Putnam et al., 2014, German translation: Kristen et al., 2007). Parents were instructed not to interact with their children during testing. They were encouraged, however, if it appeared necessary, to comfort the infant. Measures of the IBQ were not of interest for the current study and were only used for distracting the parents as they were to be tested on the same paradigm. We adapted the spatial cueing paradigm from Bertenthal et al. (2014). Figure 3 shows the sequence of a single trial of this paradigm. At the beginning of each trial, the gaze-contingent attention-getter appeared and stayed on the screen until the participant looked at it for $400 \mathrm{~ms}$ or the experimenter manually pressed a key to proceed. As soon as 


\section{Figure 3}

The timeline of each trial with gaze-contingency and minimum-maximum durations for each stimulus. ITI $=$ inter-trial interval. See the online article for the color version of this figure.



the attention-getter disappeared, the gaze-contingent stimulus cue appeared. Once the participant looked at the stimulus cue for $100 \mathrm{~ms}$ (SOA: stimulus onset asynchrony) or 5,000 ms elapsed, a peripheral target appeared and stayed on screen for a minimum of 2,500 ms and a maximum of 5,000 ms. If the participant looked at the target for $500 \mathrm{~ms}$ during the time interval of 2,000-5,000 $\mathrm{ms}$ or 5,000 ms elapsed, the target and the stimulus cue disappeared. Following the disappearance of the target and the stimulus cue, there was an inter-trial interval (ITI) for $34 \mathrm{~ms}$. The ITI was just a plain black screen. After the ITI a new trial began.

Subsequently, infants and their parents were assessed in a decorated room paradigm (Liszkowski \& Tomasello, 2011) and parents were assessed on a gesture proneness task 
(Feyereisen \& Havard, 1999), which we did not analyze in the present study. Then, parents proceeded to the same spatial cueing paradigm on the eye tracker that their infant already went through, where the same procedure was carried out. Meanwhile, their infant was cared for by an intern or a relative of the infant in a different room. Finally, the parent and their infant returned to the reception room, where the parent filled out a last questionnaire on children's pointing behavior, which we did not analyze in the present study. Finally, the child was given a symbolic certificate and a gift, and the parents were debriefed.

\section{Data Analysis}

Adults' and infants' data were analyzed separately. The dependent variable was the saccadic reaction time (SRT). SRT is defined as the time it takes to move the gaze to the target once the target has appeared (i.e. the participant fixated on the stimulus cue for at least $100 \mathrm{~ms}$ or the stimulus cue stayed on the screen for 5,000 ms). To measure the SRT, we analyzed the start time of the first fixation within the target AOI after the appearance of the target. Based on Bertenthal et al. (2014), we excluded SRTs shorter than 200 ms and longer than 3,000 ms from our analysis of the infants and based on Munoz et al. (1998), we excluded SRTs shorter than $90 \mathrm{~ms}$ and longer than 1,000 ms from our analysis of the adults.

First, we ran a separate linear mixed model each for infants' and adults' data, regressing trial-wise SRTs on hand shape, congruency, and their interaction as fixed effects, and participant (infant, adult) and trial as random effects (random intercepts). We included trials as a random effect in our model based on Bertenthal et al. (2014). We aimed for a maximal random effects structure by including random slopes defined for the congruency-hand interaction in our models that was intended to capture the individual differences across participants, but eventually removed it due to the models' lack of convergence (Barr et al., 2013). We identified the pinky finger as the reference level for the hand shape variable because it was expected to result in the smallest cueing effect, if at all present. To test for differences between congruent and incongruent trials within specific 
hand shapes, we ran planned contrasts per hand shape and congruency (e.g., index finger congruent vs. index finger incongruent). We initially pre-registered to run two repeated-measures 3 (hand shape) x 2 (congruency) ANOVAs on infants' and adults' mean SRTs. However, the model assumptions for the ANOVA were not met. Since mixed-linear models are robust against violations of model assumptions (Schielzeth et al., 2020), we report the results of the mixed-linear models. The results of the ANOVAs can be found in the Appendix.

To further support our mixed effects analyses, we ran Friedman tests on infants' and adults' proportion of positive and negative cueing effects per hand shape. For each participant and each hand shape, we calculated cueing effects by subtracting the mean SRT for congruent trials from the mean SRT for the incongruent trials. A positive cueing effect indicates that the participants reacted faster to congruent than to incongruent trials. A negative cueing effect indicates that participants reacted faster to the incongruent than to the congruent trials. To test for differences between hand shapes, we ran Holm-corrected post-hoc tests.

Furthermore, we exploratorily tested whether adults' and their infants' cueing effect and general processing speed were related. We ran Spearman correlations on adults' and infants' cueing effects for each hand shape. Furthermore, we ran Spearman correlations between adults' and infants' SRTs averaged across congruent and incongruent trials for each hand shape.

Results

The average number of trials completed was $84.1(S D=14.6$, Range $=[44 ; 96])$

for infants (congruent: $M=42.4, S D=7.2$, Range $=[24 ; 48]$; incongruent: $M=41.7$, $S D=7.6$, Range $=[20 ; 48])$ and $91.4(S D=5.8$, Range $=[67 ; 96])$ for parents (congruent: $M=46.1, S D=2.5$, Range $=[36 ; 48]$; incongruent: Mean $=45.3$, $S D=3.7$, Range $=[30 ; 48])$. See Table 1 for the number of completed trials, SRT and 
cueing effect values for each hand shape and congruency condition, for both infants and adults. For both infants and adults, the number of completed trials did not significantly differ between the congruent and incongruent conditions for any of the hand shapes (all Holm-adjusted $p \mathrm{~s}>.128)$, and between the hand shapes for either congruency condition (all Holm-adjusted $p \mathrm{~s}=1$ ).

\section{Infants' Results}

A preliminary linear mixed model on infants' data, incorporating infants' sex into the hand shape and congruency interaction, revealed neither a significant 3-way interaction $(p=.491)$ nor a significant main effect of sex $(p=.264)$. Hence, sex was not further included in the final model. The summary of the final model on infants' SRTs is shown in Table 2. An ANOVA run on the model revealed a main effect for congruency $(F(1,1624)$ $=5.14, p=.023)$, no main effect of hand shape $(F(2,2666)=0.24, p=.785)$, and a significant interaction of congruency and hand shape $(F(2,2614)=3.12, p=.044)$. The planned contrasts revealed a significant difference between congruent and incongruent trials for the whole hand (Estimate $=-49.97, S E=16.7, p=.008$ ), but not for the index finger $($ Estimate $=7.78, S E=16.4, p=.952)$, or the pinky finger $($ Estimate $=-23.97$, $S E=16.7, p=.387)$. Infants reacted faster to congruent than incongruent trials only for the whole hand but not for the index finger or the pinky finger (Figure 4).

The Friedman test on the proportion of positive and negative cueing effects yielded a significant effect, $X^{2}(5)=29.42, p<.001$. The post-hoc tests revealed a significant difference between positive and negative cueing effects for the whole hand $(p<.001)$, but not the index finger or the pinky finger (both $p \mathrm{~s}>.741$ ). For the whole hand, infants more often showed a positive than a negative cueing effect.

\section{Adults' Results}

The summary of the linear mixed model on adults' SRTs is shown in Table 2. An ANOVA run on the model revealed a main effect for congruency $(F(1,3650)=456.03, p$ 


\section{Table 1}

Number of completed trials, SRT and cueing effect per hand shape and congruency. We report mean, standard deviation, and the range (minimum and maximum).

\begin{tabular}{|c|c|c|c|}
\hline Hand form & Number of trials & SRT & Cueing effect \\
\hline Congruency & $\mathrm{M}(\mathrm{SD})$ [Range] & $\mathrm{M}$ (SD) [Range] & $\mathrm{M}$ (SD) [Range] \\
\hline \multicolumn{4}{|c|}{ Infants } \\
\hline Index finger & & & $-8.4(113)[-339 ; 217]$ \\
\hline Congruent & $14.0(2.5)[8 ; 16]$ & 469 (121) [298; 839] & \\
\hline Incongruent & $13.9(2.6)[7 ; 16]$ & $461(123)[304 ; 841]$ & \\
\hline Whole hand & & & $53.5(133)[-364 ; 460]$ \\
\hline Congruent & $14.2(2.4)[8 ; 16]$ & 429 (128) [293; 788] & \\
\hline Incongruent & $13.8(2.9)[6 ; 16]$ & $483(150)[282 ; 1069]$ & \\
\hline Pinky finger & & & $25.1(145)[-347 ; 575]$ \\
\hline Congruent & $14.2(2.6)[7 ; 16]$ & $460(130)[295 ; 778]$ & \\
\hline Incongruent & $13.9(2.5)[7 ; 16]$ & $486(154)[297 ; 1052]$ & \\
\hline \multicolumn{4}{|c|}{ Adults } \\
\hline Index finger & & & $93.4(58.1)[-9 ; 316]$ \\
\hline Congruent & $15.5(1.0)[13 ; 16]$ & $268(63)[175 ; 517]$ & \\
\hline Incongruent & $15.1(1.3)[10 ; 16]$ & $361(84)[223 ; 575]$ & \\
\hline Whole hand & & & $70.5(62.8)[-141 ; 217]$ \\
\hline Congruent & $15.4(0.9)[13 ; 16]$ & $272(67)[170 ; 573]$ & \\
\hline Incongruent & $15.0(1.6)[9 ; 16]$ & $342(70)[243 ; 527]$ & \\
\hline Pinky finger & & & $82.5(59.9)[-20 ; 302]$ \\
\hline Congruent & $15.3(1.2)[10 ; 16]$ & $270(66)[182 ; 565]$ & \\
\hline Incongruent & $15.1(1.3)[10 ; 16]$ & 353 (79) [244; 616] & \\
\hline
\end{tabular}









\section{Figure 4}

Predicted values of SRTs for infants and adults

$$
\text { Congruency } \multimap \text { Congruent } \multimap-\text { Incongruent }
$$

\section{Infants}

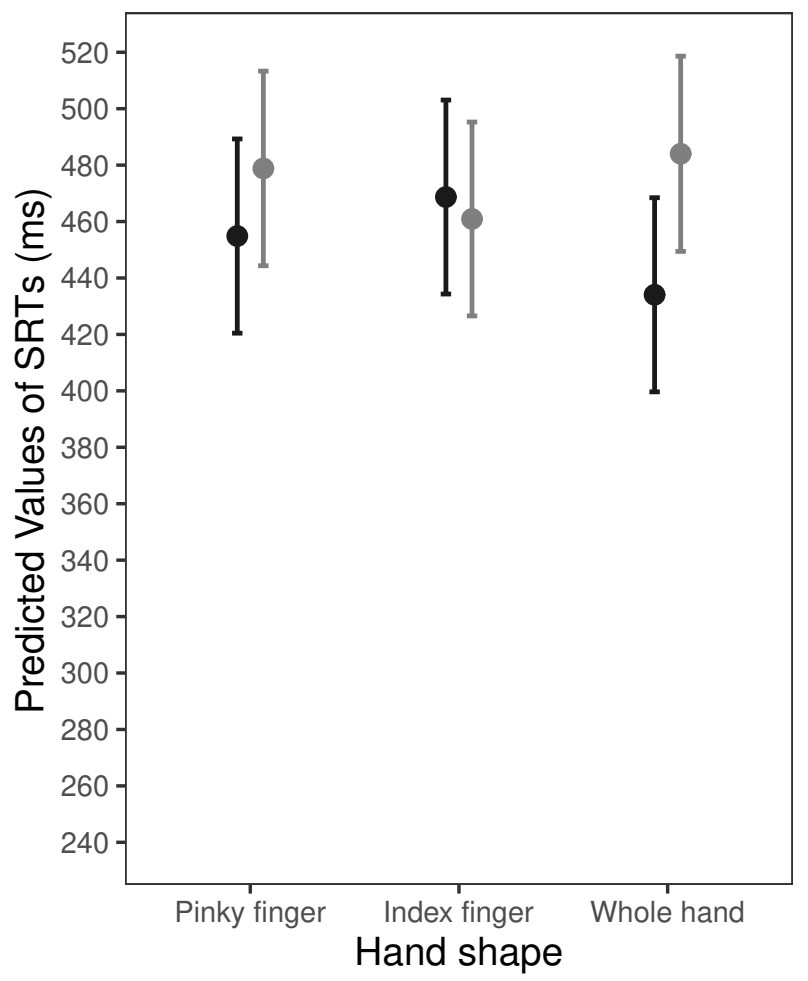

Adults

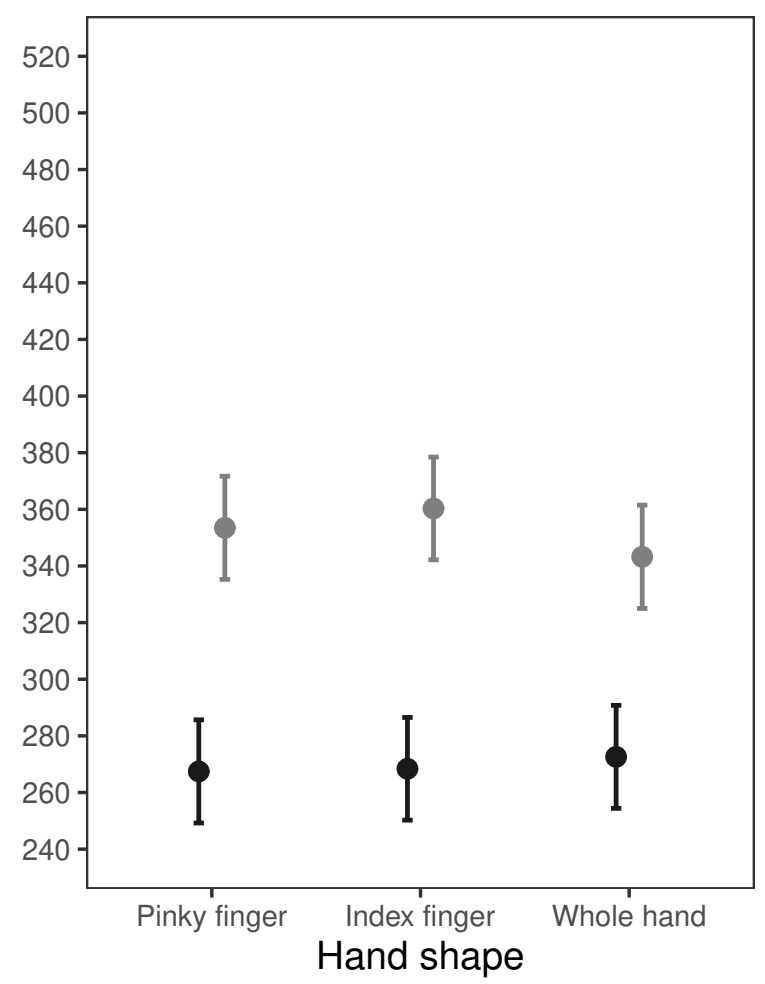

$<$.001), but no main effect of hand shape $(F(2,4724)=.99, p=0.370)$, and no significant interaction of congruency and hand shape $(F(2,4683)=2.93, p=.054)$. The planned contrasts revealed a significant difference between congruent and incongruent trials for the whole hand (Estimate $=-70.70, S E=6.62, p<.001$ ), the index finger (Estimate $=-91.90, S E=6.40, p<.001)$, and the pinky finger $($ Estimate $=-86.0, S E=6.65, p$ $<$.001). For all the hand shapes, adults showed a reliable cueing effect and reacted faster to the congruent trials than the incongruent trials (Figure 4). The cueing effect did not differ between the hand shapes.

The Friedman test on the proportion of positive and negative cueing effects yielded a significant effect, $X^{2}(5)=247.28, p<.001$. The post-hoc tests revealed a significant 
difference between positive and negative cueing effects for all hand shapes (all $p \mathrm{~s}<.001$ ). Adults more often showed a positive than a negative cueing effect for all hand shapes.

\section{Correlation of Infants' and Adults' Data}

Since the distribution of data was not normal for at least one of the variables, non-parametric Spearman correlations were conducted for the following analyses. Note that the sample size in these analyses is 54 because the data of one infant's parent was excluded due to not completing the minimum required number of trials. Adults' and infants' cueing effects significantly, but negatively, correlated for the pinky finger, $r(52)$ $=-.31, p=.025$. There were no significant correlations for the index finger, $r(52)=-.10$, $p=.459$, or the whole hand, $r(52)=-.23, p=.098$.

Adults' and infants' mean SRTs averaged across congruent and incongruent trials were not significantly correlated for either the whole hand, $r(52)=.02, p=.908$, the index finger, $r(52)=-.23, p=.092$, or the pinky finger, $r(52)=-.15, p=.272$.

\section{Discussion}

In this study, we investigated whether and to what extent adults and 12-month-old infants follow pointing gestures using the index finger, whole hand, and the pinky finger. We assessed the pointing perception with a spatial cueing paradigm with different hand shapes as cues. Adults showed a cueing effect for all hand shapes, with no difference in the extent of the cueing effect between the hand shapes. Infants showed a cueing effect only for the whole hand.

Partly in line with our hypothesis, adults showed a cueing effect for all the hand shapes presented. This replicates previous findings which used manual responses (Amano et al., 2004; Nishimura \& Michimata, 2013), by using gaze responses. It further extends previous findings by showing that adults also regard the whole hand and the pinky finger as attention-directing gestures. However, contrary to the previous findings and our 
expectation, adults did not show a larger cueing effect by the index-finger pointing. This discrepancy might have to do with at least two methodological aspects.

First, slight differences exist in the stimulus onset asynchronies (SOAs) used in spatial cueing tasks to assess pointing perception. Previous studies found a larger cueing effect for the index-finger shape at an SOA of $107 \mathrm{~ms}$ (Ariga \& Watanabe, 2009) and 160 ms (Nishimura \& Michimata, 2013), while the present study used an SOA of $100 \mathrm{~ms}$ following Bertenthal et al. (2014). Although a difference of $7 \mathrm{~ms}$ might sound trivial, it might be crucial in the context of spatial cueing which requires the participants to sufficiently process the information in the central stimulus cue to orient their attention. In fact, one study by Daum and Gredebäck (2011b) found a cueing effect in adults by an index-finger pointing only at an SOA of $300 \mathrm{~ms}$ but not $100 \mathrm{~ms}$ (but see Aldaqre et al., 2016). Hence, increased sensitivity to the social-communicative nature of the conventional index-finger pointing gesture may only emerge at an SOA that allows for sufficient processing of this symbolic information. In other words, it appears that the larger the SOA, the more time to process the social/functional meaning of the index finger, and in turn, the larger the cueing effect for the index-finger pointing.

Second, with short SOAs, the cueing effect by manual responses seems to be larger than gaze responses (Briand et al., 2000; Khatoon et al., 2002). This might be another reason why the present study did not observe a larger cueing effect for the index-finger pointing with a gaze response as opposed to the previous research that used manual responses. Future studies can test adults with a paradigm that incorporates a simultaneous measure of eye movements and manual key presses and use different SOAs to determine whether the modality of response and the time to process the stimulus cue impact the attentional shifts cued by pointing gestures of different hand shapes.

It should also be noted that, as one limitation due to the nature of the present study in which we tested the caregivers of infants as our adult sample, all but three of the adult participants were female, and all were parents. Neither do we expect a gender difference in 
adults' pointing perception based on previous studies, which do not report a gender effect cf. Ariga and Watanabe, 2009; Nishimura and Michimata, 2013, nor do we have any reason to expect parents to show a different pattern in pointing perception than non-parents. Still, a worthy future direction is to replicate these findings with a balanced sample of male and female adults, and parent and non-parents, to increase the external validity of the findings.

Contrary to our expectations, the infants showed a cueing effect only for the

whole-hand pointing. In the first year of life, infants usually start pointing with their whole hands which only gradually lends itself to pointing with the index finger (Carpendale \& Carpendale, 2010; Cochet \& Vauclair, 2010; Ger et al., 2018; Lock et al., 1990). At 12 months, infants are documented to produce both index-finger and whole-hand pointing (Ger et al., 2018; Liszkowski \& Tomasello, 2011; Lüke, Ritterfeld, et al., 2017). Hence, this current finding partially supports the following two proposals: Firstly, infants' perception of goal-directed actions might be related to their production of these actions (Ambrosini et al., 2013; Daum et al., 2013; Gampe et al., 2015; Melzer et al., 2012; Sommerville et al., 2005; Wermelinger et al., 2018). Secondly, they might be learning to follow pointing through the feedback they receive from adults for their own pointing (Leung \& Rheingold, 1981; Moore \& Corkum, 1994). It only partially supports these ideas because, under these accounts, we expected infants to show an equal cueing effect for the index finger and whole hand and show a weaker or no cueing effect for the pinky finger. However, while showing a cueing effect for the whole hand and not showing a cueing effect for the pinky finger, infants failed to show a cueing for the index finger. Nonetheless, under the proposal that infants learn to follow pointing through the feedback they receive from adults for their own pointing, it is plausible that infants' own pointing behavior prior to 12 months of age will be more determinant for their point-following at 12 months. Because, infants will need some time and experience for learning the relation between their own pointing and receiving caregiver feedback. Given that before 12 months infants point with a larger rate of whole-hand points (Carpendale \& Carpendale, 2010; Cochet \& Vauclair, 2010; Ger 
et al., 2018; Lock et al., 1990), and caregivers are equally responsive to their child's whole-hand and index-finger points (Ger et al., 2018), it is still likely that infants learn the association between pointing and others' responses better for the whole hand, and thus show a cueing effect only for the whole hand. In other words, infants might make use of their own production experience with whole-hand pointing, which is presumably greater than their experience with index-finger pointing. Accordingly, they can use this increased experience to feed their pointing perception.

Another possible interpretation for this pattern, which we did not introduce earlier, is that pointing originates from reaching (Leung \& Rheingold, 1981; Murphy \& Messer, 1977). According to this view, infants' reaching, which is initially an instrumental action, is gradually socialized by caregiver responses into a referential pointing gesture. Although we did not use a grasping hand cue in the present study, the whole hand may approximate a reaching action compared with the index- or pinky-finger shapes. A plethora of research shows that infants are cued by a grasping hand cue well before 12 months (e.g., Daum \& Gredebäck, 2011a). In that respect, at the earlier stages of their communicative development infants may focus more globally on the direction of a hand - potentially indicating a target to grasp - before they eventually home in on the social-communicative relevance of symbolic gestures such as the index-finger pointing and focus on the referent of the pointing gesture. This possibility would also closely relate with the fact that the pointing cues used in the current paradigm are rather disembodied and therefore may lack the essential social-communicative features of real human pointing and signal rather instrumental actions. In turn, in the spatial cueing context that we employed in our study, the pointing gestures in either hand shape might have failed to mirror a real referential pointing instance without supporting social-communicative cues like human speech. For example, Daum et al. (2013) found that 12-month-old infants robustly oriented to an index-finger pointing cue only when it was accompanied by human speech but not backward speech, artificial sound or no sound. If infants in our study interpreted all pointing shapes 
as instrumental, then it is conceivable that they oriented better to whole-hand pointing as the shape that most closely resembles an instrumental action, namely grasping.

We adapted our task from Bertenthal et al. (2014), who in fact found a cueing effect of index-finger pointing already with 4- and 6-month-old infants. Although we used the exact same design (with $100 \mathrm{~ms} \mathrm{SOA}$ ) and the same attention-getter and target stimuli, we did not find a cueing effect for the index-finger pointing with 12-month-old infants. What could account for this discrepancy? One possibility concerns the difference in the orientation of the pointing hand in our task. While the palm of the hand was facing downwards in Bertenthal et al. (2014), it was facing the viewer in our task, to be better able to illustrate the whole-hand pointing. Nevertheless, this is not likely to be the cause, because Rohlfing et al. (2012) found a cueing effect of the index-finger pointing with 4.5and 6.5-month-old infants using the same orientation of the hand as we did. A second possible explanation could lie in the within-subject design we used where all infants saw all hand shapes as cues. That is, the whole hand shape might have biased infants' attention as the more salient cue and might have masked any congruency effects that could have emerged by the index- or pinky-finger pointing if they had been presented alone. Apart from methodological differences, a third possibility concerns the developmental progress in the processing of pointing gestures. Previous research seems to demonstrate generally larger cueing effects at younger ages in the first year (Bertenthal et al., 2014; Rohlfing et al., 2012), which progressively becomes more fine-tuned to the social-communicative nature of the pointing gestures and approach adult-like processing toward the end of the first year (Daum et al., 2013; Melinder et al., 2015). Hence, infants may go through a development whereby they transition from a more bottom-up, reflexive orienting to attention-directing nature of pointing stimuli, to a more top-down processing that incorporates the symbolic and social-communicative meaning of the pointing stimuli. Infants' own pointing behavior may be one of the drivers of this transition, whereby they appreciate the symbolic and social-communicative meaning of the pointing the more they produce pointing themselves 
and consequently initiate episodes of joint attention with interaction partners.

Sensitivity towards the index-finger pointing might still be fragile early in development and completely tuning in to this canonical shape might take a while. For example, at 6 months, infants failed to orient their attention to the pointed target if the pointing hand and finger were not directed in the same direction as the arm (Schmitow et al., 2016). Similarly, 2-year-old, but not 3-year-old, children have been shown to fail to search an object in the direction of an index-finger pointing in elbow cross-pointing, where the elbow was directed to the opposite direction of the index finger (Lakatos et al., 2009). Hence, young children seem to still focus on the salient features of directionality until they can focus their attention completely on the conventional signs of their society.

In fact, sensitivity to the canonical index-finger pointing in the perception of pointing might need to await infants' pointing predominantly with the index finger, which seems to develop around 16 months (Lüke, Ritterfeld, et al., 2017; Ramos-Cabo et al., 2020). Thus, a future direction is to test infants at or older than 16 months of age to quest for sensitivity to the index-finger pointing. Moreover, future studies may look for direct links between infants' perception of pointing with different hand shapes, their own production of pointing with different hand shapes, and their caregivers' production of index-finger points, in order to better account for the underlying mechanism(s) for the development of pointing perception in early childhood.

Our exploratory analyses of the correlation between the infants' and their parents' mean SRTs and cueing effects revealed a significant link only in the cueing effect for the pinky finger, but surprisingly in the negative direction. The fact that there was not an overall correlation in the mean SRTs but only in the cueing effect for the pinky finger suggests that irrespective of the processing speed of the gesture stimuli, infants who were better in orienting their attention in the direction of a pinky-finger pointing had parents who were worse in this. At this point, we are not able to offer a plausible interpretation for this surprising pattern. 
In conclusion, the present study found that adults did not show increased sensitivity to the conventional index-finger pointing compared to other hand shapes. In contrast, they showed cueing effects of equal sizes for all three hand shapes tested (i.e., index finger, whole hand, pinky finger). Twelve-month-olds showed a cueing effect only for whole-hand pointing, suggesting that at least two compelling, but not necessarily mutually exclusive, mechanisms might be at play in the development of pointing perception: Infants learn to orient to pointing gestures by the responses they receive to their own pointing, and infants' production and perception of pointing gestures develop hand in hand. More research with older infants is needed to further support these claims. 
544

545

\section{Acknowledgements}

We are grateful to the parents and infants who participated in this study. Many thanks to Dr. Anja Gampe for her support in building the eye-tracking task and her suggestions for the data analyses, and Katrin Hettwer for her help in data collection. 


\section{References}

Aldaqre, I., Schuwerk, T., Daum, M. M., Sodian, B., \& Paulus, M. (2016). Sensitivity to communicative and non-communicative gestures in adolescents and adults with autism spectrum disorder: Saccadic and pupillary responses. Experimental Brain Research, 234(9), 2515-2527. https://doi.org/10.1007/s00221-016-4656-y

Amano, S., Kezuka, E., \& Yamamoto, A. (2004). Infant shifting attention from an adult's face to an adult's hand: A precursor of joint attention. Infant Behavior and Development, 27(1), 64-80. https://doi.org/10.1016/j.infbeh.2003.06.005

Ambrosini, E., Reddy, V., de Looper, A., Costantini, M., Lopez, B., \& Sinigaglia, C. (2013). Looking ahead: Anticipatory gaze and motor ability in infancy. PLoS ONE, 8(7), 1-9. https://doi.org/10.1371/journal.pone.0067916

Ariga, A., \& Watanabe, K. (2009). What is special about the index finger?: The index finger advantage in manipulating reflexive attentional shift. Japanese Psychological Research, 51(4), 258-265. https://doi.org/10.1111/j.1468-5884.2009.00408.x

Barr, D. J., Levy, R., Scheepers, C., \& Tily, H. J. (2013). Random effects structure for confirmatory hypothesis testing: Keep it maximal. Journal of Memory and Language, 68(3), 255-278. https://doi.org/10.1016/j.jml.2012.11.001

Bertenthal, B. I., Boyer, T. W., \& Harding, S. (2014). When do infants begin to follow a point? Developmental Psychology, 50(8), 2036-2048. https://doi.org/http://dx.doi.org/10.1037/a0037152

Briand, K. A., Larrison, A. L., \& Sereno, A. B. (2000). Inhibition of return in manual and saccadic response systems. Perception \&6 Psychophysics, 62(8), 1512-1524. https://doi.org/10.3758/BF03212152

Calvo-Merino, B., Grèzes, J., Glaser, D. E., Passingham, R. E., \& Haggard, P. (2006). Seeing or Doing? Influence of Visual and Motor Familiarity in Action Observation. Current Biology, 16(19), 1905-1910. https://doi.org/10.1016/j.cub.2006.07.065 
Camaioni, L., Perucchini, P., Bellagamba, F., \& Colonnesi, C. (2004). The role of declarative pointing in developing a theory of mind. Infancy, 5(3), 291-308. https://doi.org/10.1207/s15327078in0503_3

Cameron-Faulkner, T., Theakston, A., Lieven, E., \& Tomasello, M. (2015). The relationship between infant holdout and gives, and pointing. Infancy, 20(5), 576-586. https://doi.org/10.1111/infa.12085

Carpendale, J. I., \& Carpendale, A. B. (2010). The development of pointing: From personal directedness to interpersonal direction. Human Development, 53(3), 110-126. https://doi.org/10.1159/000315168

Carpenter, M., Nagell, K., Tomasello, M., Butterworth, G., \& Moore, C. (1998). Social Cognition, Joint Attention, and Communicative Competence from 9 to 15 Months of Age. Monographs of the Society for Research in Child Development, 63(4), i-174. https://doi.org/10.2307/1166214

Cochet, H., \& Vauclair, J. (2010). Pointing gesture in young children: Hand preference and language development. Gesture, 10(2-3), 129-149. https://doi.org/10.1075/gest.10.2-3.02coc

Cochet, H., \& Vauclair, J. (2014). Deictic gestures and symbolic gestures produced by adults in an experimental context: Hand shapes and hand preferences. Laterality, 19(3), 278-301. https://doi.org/10.1080/1357650X.2013.804079

Daum, M. M., \& Gredebäck, G. (2011a). The development of grasping comprehension in infancy: Covert shifts of attention caused by referential actions. Experimental Brain Research, 208(2), 297-307. https://doi.org/10.1007/s00221-010-2479-9

Daum, M. M., \& Gredebäck, G. (2011b). Spatial cueing by referential human gestures, arrows and mechanical devices. International Journal of Mind, Brain and Cognition, 2, 113-126. Retrieved April 8, 2021, from https://www.zora.uzh.ch/id/eprint/81135/ 
Daum, M. M., Prinz, W., \& Aschersleben, G. (2011). Perception and production of object-related grasping in 6-month-olds. Journal of Experimental Child Psychology, 108(4), 810-818. https://doi.org/10.1016/j.jecp.2010.10.003

Daum, M. M., Ulber, J., \& Gredebäck, G. (2013). The development of pointing perception in infancy: Effects of communicative signals on covert shifts of attention. Developmental Psychology, 49(10), 1898-1908. https://doi.org/10.1037/a0031111

Deák, G. O., Flom, R. A., \& Pick, A. D. (2000). Effects of gesture and target on 12- and 18-month-olds' joint visual attention to objects in front of or behind them. Developmental Psychology, 36(4), 511-523. https://doi.org/10.1037/0012-1649.36.4.511

Diersch, N., Cross, E. S., Stadler, W., Schütz-Bosbach, S., \& Rieger, M. (2012). Representing others' actions: The role of expertise in the aging mind. Psychological Research, 76 (4), 525-541. https://doi.org/10.1007/s00426-011-0404-x

Feyereisen, P., \& Havard, I. (1999). Mental imagery and production of hand gestures while speaking in younger and older adults. Journal of Nonverbal Behavior, 23(2), 153-171. https://doi.org/10.1023/A:1021487510204

Flack, Z. M., Naylor, M., \& Leavens, D. A. (2018). Pointing to visible and invisible targets. Journal of Nonverbal Behavior, 42(2), 221-236. https://doi.org/10.1007/s10919-017-0270-3

Gampe, A., Prinz, W., \& Daum, M. M. (2015). Measuring action understanding: Relations between goal prediction and imitation. British Journal of Developmental Psychology, 1-13. https://doi.org/10.1111/bjdp.12125

Ger, E., Altınok, N., Liszkowski, U., \& Küntay, A. C. (2018). Development of infant pointing from 10 to 12 months: The role of relevant caregiver responsiveness. Infancy, 23(5), 708-729. https://doi.org/10.1111/infa.12239 
Gredebäck, G., Melinder, A., \& Daum, M. (2010). The development and neural basis of pointing comprehension. Social Neuroscience, 5(5-6), 441-450. https://doi.org/10.1080/17470910903523327

Iverson, J. M., Tencer, H. L., Lany, J., \& Goldin-Meadow, S. (2000). The relation between gesture and speech in congenitally blind and sighted language-learners. Journal of Nonverbal Behavior, 24, 105-130. https://doi.org/10.1023/A:1006605912965

Khatoon, S., Briand, K. A., \& Sereno, A. B. (2002). The role of response in spatial attention: Direct versus indirect stimulus-response mappings. Vision Research, 42(24), 2693-2708. https://doi.org/10.1016/S0042-6989(02)00327-9

Kita, S. (2003). Interplay of gaze, hand, torso orientation, and language in pointing. In S. Kita (Ed.), Pointing: Where language, culture, and cognition meet (pp. 307-328). Mahwah, NJ: Lawrence Erlbaum Associates.

Kristen, S., Eisenbeis, H., Thoermer, C., \& Sodian, B. (2007). Temperamentsfragebogen für babys-revidierte form (Unpublished).

Lakatos, G., Soproni, K., Dóka, A., \& Miklósi, Á. (2009). A comparative approach to dogs' (Canis familiaris) and human infants' comprehension of various forms of pointing gestures. Animal Cognition, 12, 621-631. https://doi.org/10.1007/s10071-009-0221-4

Leung, E. H., \& Rheingold, H. L. (1981). Development of pointing as a social gesture. Developmental Psychology, 17(2), 215-220. https://doi.org/10.1037/0012-1649.17.2.215

Liszkowski, U., \& Tomasello, M. (2011). Individual differences in social, cognitive, and morphological aspects of infant pointing. Cognitive Development, 26(1), 16-29. https://doi.org/10.1016/j.cogdev.2010.10.001

Lock, A., Young, A., Service, V., \& Chandler, P. (1990). Some observations on the origins of the pointing gesture. Springer series in language and communication (pp. 42-55). Springer Berlin Heidelberg. https://doi.org/10.1007/978-3-642-74859-2_5 
Lüke, C., Grimminger, A., Rohlfing, K. J., Liszkowski, U., \& Ritterfeld, U. (2017). In infants' hands: Identification of preverbal infants at risk for primary language delay. Child Development, 88(2), 484-492. https://doi.org/10.1111/cdev.12610

Lüke, C., Leinweber, J., \& Ritterfeld, U. (2019). Walking, pointing, talking - the predictive value of early walking and pointing behavior for later language skills. Journal of Child Language, 46(6), 1228-1237. https://doi.org/10.1017/S0305000919000394

Lüke, C., Ritterfeld, U., Grimminger, A., Liszkowski, U., \& Rohlfing, K. J. (2017). Development of pointing gestures in children with typical and delayed language acquisition. Journal of Speech, Language, and Hearing Research, 60(11), 3185-3197. https://doi.org/10.1044/2017_jslhr-l-16-0129

Matthews, D., Behne, T., Lieven, E., \& Tomasello, M. (2012). Origins of the human pointing gesture: A training study. Developmental Science, 15(6), 817-829. https://doi.org/10.1111/j.1467-7687.2012.01181.x

Melinder, A. M. D., Konijnenberg, C., Hermansen, T., Daum, M. M., \& Gredebäck, G. (2015). The developmental trajectory of pointing perception in the first year of life. Experimental Brain Research, $233(2), 641-647$. https://doi.org/10.1007/s00221-014-4143-2

Melzer, A., Prinz, W., \& Daum, M. M. (2012). Production and perception of contralateral reaching: A close link by 12 months of age. Infant Behavior and Development, 35 (3), 570-579. https://doi.org/10.1016/j.infbeh.2012.05.003

Moore, C., \& Corkum, V. (1994). Social understanding at the end of the first year of life. Developmental Review, 14(4), 349-372. https://doi.org/10.1006/drev.1994.1014

Morissette, P., Ricard, M., \& Décarie, T. G. (1995). Joint visual attention and pointing in infancy: A longitudinal study of comprehension. British Journal of Developmental Psychology, 13(2), 163-175. https://doi.org/10.1111/j.2044-835X.1995.tb00671.x 
Munoz, D. P., Broughton, J. R., Goldring, J. E., \& Armstrong, I. T. (1998). Age-related performance of human subjects on saccadic eye movement tasks. Experimental Brain Research, 121(4), 391-400. https://doi.org/10.1007/s002210050473

Murphy, C. M., \& Messer, D. J. (1977). Mothers, infants and pointing: A study of a gesture. In H. R. Schaffer (Ed.), Studies in mother-infant interaction. New York: Academic.

Nishimura, A., \& Michimata, C. (2013). Pointing hand stimuli induce spatial compatibility effects and effector priming. Frontiers in Psychology, 4, 219. https://doi.org/10.3389/fpsyg.2013.00219

Putnam, S. P., Helbig, A. L., Gartstein, M. A., Rothbart, M. K., \& Leerkes, E. (2014). Development and assessment of short and very short forms of the Infant Behavior Questionnaire-Revised. Journal of Personality Assessment, 96 (4), 445-458. https://doi.org/10.1080/00223891.2013.841171

R Core Team. (2021). R: A language and environment for statistical computing. $\mathrm{R}$ Foundation for Statistical Computing. Vienna, Austria. https://www.R-project.org/

Ramos-Cabo, S., Vulchanov, V., \& Vulchanova, M. (2020). Different ways of making a point: A study of gestural communication in typical and atypical early development. Autism Research, 00(1-13). https://doi.org/https://doi.org/10.1002/aur.2438

Rohlfing, K. J., Longo, M. R., \& Bertenthal, B. I. (2012). Dynamic pointing triggers shifts of visual attention in young infants: Dynamic pointing triggers shifts of visual attention. Developmental Science, 15(3), 426-435. https://doi.org/10.1111/j.1467-7687.2012.01139.x

Rowe, M. L., \& Goldin-Meadow, S. (2009). Differences in early gesture explain SES disparities in child vocabulary size at school entry. Science, 323(5916), 951-953. https://doi.org/10.1126/science.1167025

Rowe, M. L., \& Leech, K. A. (2019). A parent intervention with a growth mindset approach improves children's early gesture and vocabulary development. Developmental Science, 22(4), e12792. https://doi.org/10.1111/desc.12792 
Rüther, J. N. (2019). Sociocultural influences on the development of infants' cultural learning skills in the first two years of life (Doctoral dissertation). University of Hamburg. Retrieved March 29, 2021, from https://ediss.sub.uni-hamburg.de/handle/ediss/6238

Salomo, D., \& Liszkowski, U. (2013). Sociocultural Settings Influence the Emergence of Prelinguistic Deictic Gestures. Child Development, 84(4), 1296-1307. https://doi.org/https://doi.org/10.1111/cdev.12026

Schielzeth, H., Dingemanse, N. J., Nakagawa, S., Westneat, D. F., Allegue, H., Teplitsky, C., Réale, D., Dochtermann, N. A., Garamszegi, L. Z., \& Araya-Ajoy, Y. G. (2020). Robustness of linear mixed-effects models to violations of distributional assumptions. Methods in Ecology and Evolution, 11(9), 1141-1152. https://doi.org/https://doi.org/10.1111/2041-210X.13434

Schmitow, C., Kochukhova, O., \& Nyström, P. (2016). Social perception: How do 6-month-old infants look at pointing gestures? Infant Behavior and Development, 42, 152-156. https://doi.org/10.1016/j.infbeh.2015.12.001

Sommerville, J. A., Woodward, A., \& Needham, A. (2005). Action experience alters 3-month-old infants' perception of others' actions. Cognition, 96(1), B1-B11. https://doi.org/10.1016/j.cognition.2004.07.004

von Hofsten, C., Dahlström, E., \& Fredriksson, Y. (2005). 12-month-old infants' perception of attention direction in static video images. Infancy, 8(3), 217-231.

Wermelinger, S., Gampe, A., \& Daum, M. M. (2018). The dynamics of the interrelation of perception and action across the life span. Psychological Research. https://doi.org/10.1007/s00426-018-1058-8 


\section{Appendix}

First we ran separate paired t-tests for both infant's and adults' data to determine whether pointing direction influenced the mean SRTs. Results indicated no influence of pointing direction for both children $(t(54)=1.68, p=.099$, Cohen's $\mathrm{d}=.22)$ and adults $(t(63)=0.73, p=.467$, Cohen's d $=.09)$.

Next, we ran two repeated-measures 3 (hand shape) x 2 (congruency) ANOVAs on infants' and adults' mean SRTs. The analyses of the infants indicated no main effects of congruency $\left(F(1,54)=3.62, p=.063,, \eta_{p}^{2}=.06\right)$ and hand shape $(F(2,108)=0.65, p$ $\left.=.526, \eta_{p}^{2}=.01\right)$, but a significant interaction of congruency and hand shape $(F(2,108)$ $\left.=3.99, p=.021, \eta_{p}^{2}=.07\right)$. Post-hoc t-tests using Bonferroni correction revealed that infants showed a significant cueing effect only for the whole hand (index finger: $t(54)$ $=0.56$, adjusted $p=1$; whole hand: $t(62)=-8.9, p=.013$; pinky finger:t(62) $=-10.9$, $p=.612)$.

The analyses of the adults revealed a main effect of congruency $(F(1,62)=192.60$, $\left.p<.001,, \eta_{p}^{2}=.76\right)$, no effect of hand shape $\left(F(2,124)=1.60, p=.206, \eta_{p}^{2}=.03\right)$, but a significant interaction of congruency and hand shape $\left(F(2,124)=3.86, p=.024, \eta_{p}^{2}\right.$ $=.06)$. Post-hoc t-tests using Bonferroni correction revealed that adults showed a significant cueing effect for all hand shapes (index finger: $t(62)=-12.7$, adjusted $p<$ .0001 ; whole hand: $t(62)=-8.9, p<.0001$; pinky finger: $t(62)=-10.9, p<.0001)$. 